\title{
Modeling and Experiment of Leading Edge Separation Control Using SDBD Plasma Actuators
}

\author{
Dmitriy M. Orlov* \\ United States Air Force Academy \\ Space Physics and Atmospheric Research Center \\ Department of Physics \\ Colorado Springs, CO 80840 \\ Thomas Apker, ${ }^{\dagger}$ Chuan He, ${ }^{\ddagger}$ Hesham Othman ${ }^{\S}$ and Thomas C. Corke
University of Notre Dame
Center for Flow Physics and Control
Aerospace and Mechanical Engineering Department
}

Notre Dame, IN 46556

\begin{abstract}
This work presents the study of the single-dielectric barrier discharge aerodynamic plasma actuator. To model the physics of the plasma discharge, a space-time lumpedelement circuit model was developed. The model solution compared well to some of the characteristic features of the discharge such as the dependence of the sweep velocity and maximum extent of the ionized air as functions of the applied voltage and a.c. driving frequency. The time-dependent charge distribution obtained from the model was used to provide boundary conditions to the electric field equation that was used to calculate the time dependent electric potential. The was then used to calculate the space-time distribution of the actuator body force. An application of the plasma actuators to the leading-edge separation control on the NACA 0021 airfoil was studied numerically and experimentally. The results were obtained for a range of angles of attack for uncontrolled flow, and steady and unsteady plasma actuators located at the leading edge of the airfoil. The control of the lift stall was of particular interest. Improvement in the airfoil characteristics were observed in the numerical simulations at post-stall angles of attack with the plasma actuators. The computational results corresponded very well with the experiments.
\end{abstract}

\section{Introduction}

The Single Dielectric Barrier Discharge (SDBD) plasma actuators have been successfully used in a variety of different flow control applications. These include exciting boundary layer instabilities on a sharp cone at Mach 3.5, ${ }^{1}$ lift augmentation on a wing section, ${ }^{2-5}$ low-pressure turbine blade separation control, ${ }^{6-10}$ turbine tip clearance flow control, ${ }^{11-13}$ bluff body control, ${ }^{14,15}$ drag reduction, ${ }^{16,17}$ unsteady vortex generation, ${ }^{18-21}$ and airfoil leading-edge separation control. ${ }^{22-27}$ Some of the advantages of the SDBD plasma actuators over other flow control devices are: reduced size and weight, absence of moving parts, increased reliability, inexpensiveness, high bandwidth (quick response), and low parasitic drag when not operating.

The SDBD plasma actuator behavior is primarily governed by the buildup of charge on the dielectricencapsulated electrode. When a.c. voltage is applied, a plasma discharge appears on the insulator surface

\footnotetext{
*Research Scientist, Member AIAA

$\dagger$ Ph.D. Candidate

${ }^{\ddagger}$ Ph.D. Candidate

$\S$ Research Associate

ब Clark Chair Professor, Associate Fellow AIAA
} 
above the encapsulated electrode, and directed momentum is coupled into the surrounding air. In operation, the plasma in the discharge appears on the surface of the dielectric each half-cycle of the applied AC voltage.

To the unaided eye, the plasma appears as a relatively uniform diffuse discharge, but optical measurements of the plasma indicate that it is highly structured in both space and time. The temporal nature of the actuator indicates that this plasma is indeed a dielectric barrier discharge. ${ }^{28}$ The most important feature of the SDBD is that it can sustain a large-volume discharge at atmospheric pressure without the discharge collapsing into a constricted arc.

There are three separate temporal time scales that are relevant to the SDBD process. The shortest time scale, of the order of $10^{-8}$ seconds, is associated with the initiation of the micro-discharges from the exposed electrode leading to charge redistribution on the dielectric. The second time scale is related to the operation of the plasma actuator itself. It is defined by the period of the a.c. cycle that drives the alternating current discharge. This time scale is on the order of $10^{-4} \mathrm{sec}$ (for an a.c. frequency of the order of $10 \mathrm{kHz}$ ), which is approximately $10^{4}$ times slower than the time scale of the micro-discharges. The third time scale is the one that governs the movements of the neutral fluid responding to the plasma actuator. This time scale is on the order of $10^{-2}$ seconds.

The four order of magnitude difference in time scales allows us to assume that the plasma formation and charge rearrangement processes are instantaneous. This allows us to assume that the plasma is operating in a quasi-steady regime, when the charges are rearranged in the region, so that they cancel the external electric field everywhere, except the small regions near the electrodes. This leads to the quasi-DC assumption used in modeling the plasma formation and computing the plasma body force.

\section{Space-Time Lumped-Element Circuit Model}

\section{II.A. Mathematical Formulation of Space-Time Lumped-Element Circuit Model}

It has been was shown previously ${ }^{29}$ that the electrostatic model could be used to simulate the aerodynamic plasma actuator if the volume of plasma was know for the particular applied voltage conditions. An attempt to extrapolate these results to other voltage amplitudes was not possible with this model. To account for the change in the volume of plasma with respect to the applied voltage amplitude and frequency, the lumpedelement circuit model was created, ${ }^{30,31}$ and it was shown, that the spatial lumped-element circuit model correctly described many of the characteristics of the SDBD plasma actuator. For example, it was shown that the model predicts that the power dissipated in the plasma resistive element increases with the $7 / 2$ power of the applied voltage. This correlated with the experimental results of Enloe et al. ${ }^{32}$ and Post, ${ }^{24}$ that showed that induced thrust and maximum velocity generated by the asymmetric electrode arrangement of the SDBD plasma actuator varied as $V_{a p p}^{7 / 2}$.

Despite all the advantages of the earlier model, it had a significant limitation in that the dynamics of the ionization relied on empirical coefficients that were functions of the applied voltage amplitude and frequency. Therefore the model was not able to predict the actuator dependence outside of its empirical calibration space.

The space-time lumped-element circuit model presented here is intended to model the details of of the ionization process to provide predictions of the body force for a range of parameters without the need of experimental calibration. The space-time lumped-element model follows from the previous model in that the air and dielectric are represented as a network of resistors and capacitors. The difference comes in that rather than being represented by a single parallel network, the domain over the covered electrode is divided into $N$ parallel networks, such as illustrated in Figure 1. The characteristics of each parallel network depend on its distance from the exposed electrode. For example, parallel network 1 is closest to the exposed electrode and extends the shortest distance over the covered electrode. Parallel network $N$ extends the farthest distance over the covered electrode.

Each parallel network consists of an air capacitor, a dielectric capacitor, and a plasma resistive element as in the earlier model. ${ }^{29}$ In addition to these, zener diodes were added to set the threshold voltage levels at which the plasma initiates, and to switch in the different plasma resistance values based on the current direction, namely from the exposed electrode to the dielectric, or from the dielectric to the exposed electrode, that experiments have shown to be important. The N-circuit arrangement is shown in Figure 2.

The value of the air capacitor in the $n$-th sub-circuit is based on its distance from the edge of the exposed 


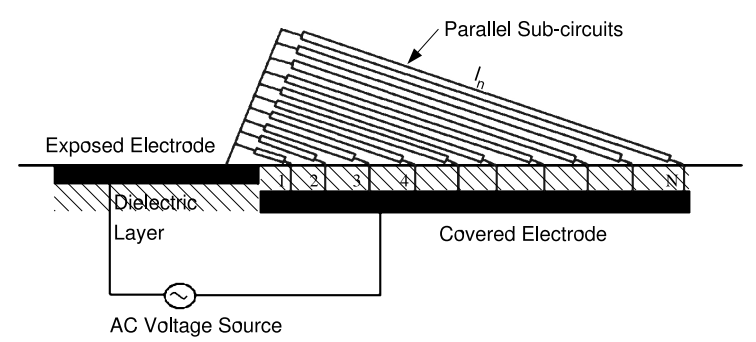

Figure 1. The physical space over the encapsulated electrode is divided into $\mathrm{N}$ sub-regions.

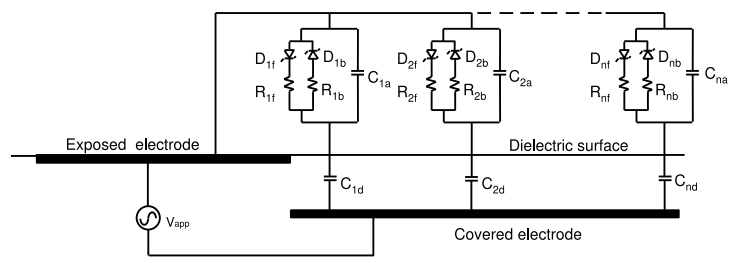

Figure 2. Electric circuit model of a single dielectric aerodynamic plasma actuator.

electrode. This is given by equation (1)

$$
C_{a n}=\frac{\varepsilon_{0} \varepsilon_{a} A_{n}}{l_{n}}
$$

where $\varepsilon_{a}$ is the dielectric coefficient of air, $l_{n}$ is the representative distance over the dielectric surface, and $A_{n}$ is the cross section of this air capacitor. The cross-section $A_{n}$ is the product of the span-wise size of the actuator, $z_{n}$, and the height of the capacitive element, $h_{n}$. The schematic of this capacitive element is shown in Figure 3.

The resistance value in the $n$-th sub-circuit is similarly based on its distance from the exposed electrode. It is given by equation (2)

$$
R_{n}=\frac{\rho_{a} l_{n}}{A_{n}}
$$

where $\rho_{a}$ is the effective resistivity of the air.

The value of the dielectric capacitor for each sub-circuit is similar to the air capacitance except that it is based on the properties of the dielectric material. It is given by equation (3)

$$
C_{d n}=\frac{\varepsilon_{0} \varepsilon_{d} A_{d}}{l_{d}}
$$

where $\varepsilon_{d}$ is the dielectric coefficient of the dielectric material, $l_{d}$ is the thickness of this material, and $A_{n}$ is the cross section which is equal to the product of the span-wise size of the actuator, $z_{n}$, and the width of the dielectric capacitive element, $d_{n}$, as shown in Figure 4.

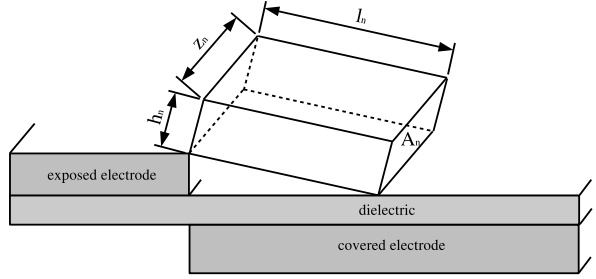

Figure 3. Schematic drawing of the n-th air capacitor.

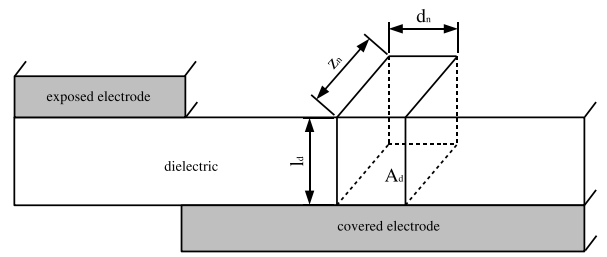

Figure 4. Schematic drawing of the n-th dielectric capacitor.

If we assume that the paths are parallel to each other, and the length of path $l_{n}$ is proportional to its position number $n$, then it follows that the the air capacitance of the $n$-th sub-circuit, $C_{a n}$, is proportional to $1 / n$, and the air resistance of the $n$-th sub-circuit is proportional to $n$. Therefore sub-circuits that are furthest from the edge of the electrodes, would have the lowest air capacitance and the largest air resistance.

For a time-varying applied voltage, the voltage on the surface of the dielectric at the $n^{t h}$ parallel network is given as

$$
\frac{d V_{n}(t)}{d t}=\frac{d V_{a p p}(t)}{d t}\left(\frac{C_{a n}}{C_{a n}+C_{d n}}\right)+k_{n} \frac{I_{p n}(t)}{C_{a n}+C_{d n}},
$$

where $I_{p n}(t)$ is the time varying current through the plasma resistor. The zener diode in the parallel network has two functions. The first is setting a threshold on the voltage differential between the exposed electrode and the dielectric surface above which the air is ionized (plasma formed). In equation (4), the diodes are represented by the variable $k_{n}$. When the threshold voltage is exceeded, $k_{n}=1$. Otherwise $k_{n}=0$. 
The second function of the zener diodes was to switch in either one of the resistances representing the plasma resistance based on the direction of the current, from the exposed electrode to the dielectric surface or vice versa. This is meant to represent the experimental observations that the current and corresponding plasma illumination were different based on the current direction. The resistance values are designated as $R_{n f}$ or $R_{n b}$ (representing forward or backward going current). The ratio of the two plasma resistances used in the simulation were $\frac{R_{n f}}{R_{n b}}=5$. This was based on the difference in the estimated current observed in experiments. With this background, the current through the plasma resistance, $I_{p}(t)$ is given as

$$
I_{p n}(t)=\frac{1}{R_{n}}\left[V_{a p p}(t)-V_{n}(t)\right]
$$

where $R_{n}=R_{n f}$ or $R_{n b}$ based on the current direction.

This space-time formulation of the lumped-element circuit model has many advantages over the previous model. One advantage is that it allows us to specify the number of the sub-circuits $N$ making up the actuator. Ideally, as $N \rightarrow \infty$, the numerical solution should approach the asymptotic solution. As the tests of the code showed, values of $N \approx 10^{2}$ appear to be sufficient to capture the essential physics.

A second advantage of this formulation is that it is temporal. Therefore the effect of the a.c. frequency or wave form can be examined. Dynamics such as the sweep-out velocity of the plasma can then be determined a priori.

\section{II.B. Results of Space-Time Lumped-Element Circuit Model}

As an example, the electric circuit equations (4) were solved for 100 parallel circuit elements making up a SDBD actuator. For this, the applied voltage was a sine wave with amplitude of $5 k V_{p-p}$, and a frequency of $5000 \mathrm{~Hz}$. In order to resolve the dynamic motions of the plasma, a computational time step of $8 \cdot 10^{-7}$ seconds was used. This corresponded to 250 time steps within each cycle of the a.c. input period.

The solution of the model equations gives the voltage on the surface of the dielectric, $V_{n}$, for each parallel circuit element. An example of this for the first five circuit elements, closest to the exposed electrode, is shown in Figure 5. We observe that there is a shift in time of the peak voltage in successive current elements. This reflects the sweep out of the plasma over the dielectric surface.

Figure 6 shows the time series in the current through the first five circuit elements in the model. Plasma is formed where the current is non-zero. This is observed to occur twice per a.c. period at the time periods when the voltage difference between the exposed electrode and dielectric surface exceeds a threshold level. Again we observe that the time when the plasma first forms increases as the sub-circuit is further from the exposed electrode. As with the voltage, this reflects the sweep out of the plasma during the input a.c. cycle.

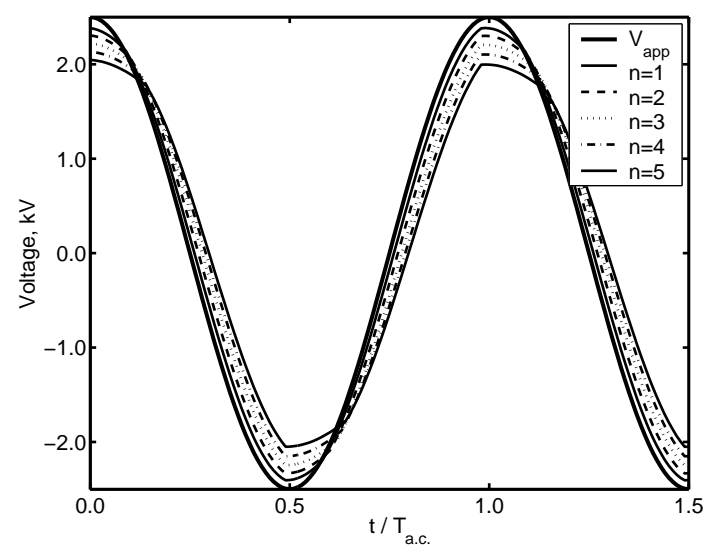

Figure 5. Voltage on the surface of the dielectric in the first five sub-circuits $(n=1,2,3,4,5)$ obtained from space-time lumped element circuit model.

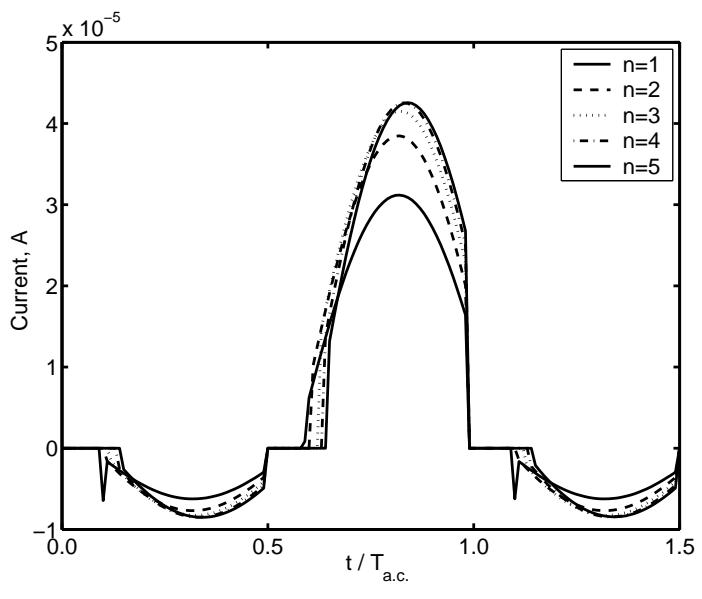

Figure 6. Plasma current in the first five subcircuits $(n=1,2,3,4,5)$ obtained from spacetime lumped element circuit model. 
As has been noted previously in the experiments, the electric current in the plasma relates directly to the light emission from the SDBD, ${ }^{29,32,33}$ and thus is an indication of the volume of the plasma. Therefore to compare to the experiments, the rectified plasma current from the model in time and space above the dielectric-covered electrode is shown in Figure 7 as contour lines of the constant plasma current. Zero on the space axis corresponds to the edge of the exposed electrode. The time axis is shown as a fraction of the input a.c. period. This can be directly compared to Figure 8 that shows the space-time variation of the plasma illumination.

The rectified current from the model is very similar to the experimental result. It clearly shows the largest current at the edge of the exposed electrode and the rapid decay when moving away from electrode's edge. In addition, the model simulates ionization occurring twice during the a.c. cycle, and the difference in the magnitude of the current in the two halves of the cycle. This again illustrates the good agreement between the model and the photo-multiplier experiments.

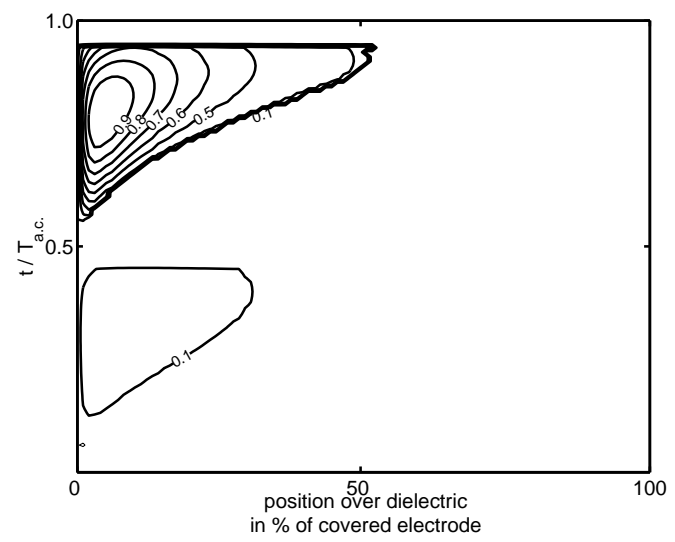

Figure 7. Contour lines of constant rectified plasma current obtained from space-time lumped element circuit model.

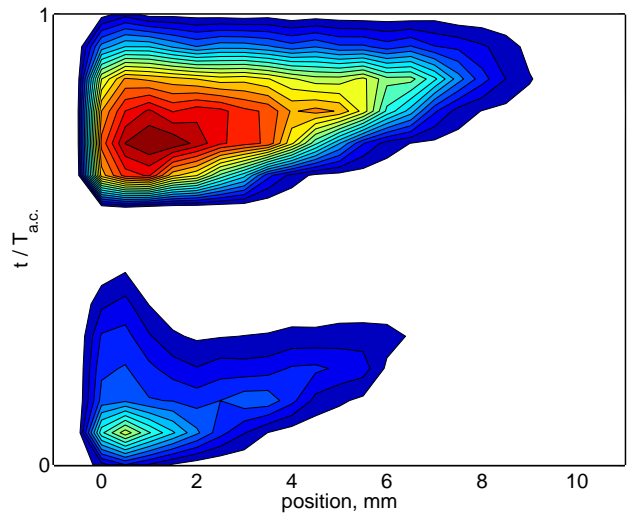

Figure 8. Contour lines of space-time variation of the measured plasma light emission for SDBD plasma actuator corresponding to one period, $T$, of the input a.c. cycle.

The results from the photo-multiplier experiments and from the space-time lumped-element circuit model can be compared quantitatively. There are two important characteristics of the plasma discharge that can be extracted from this data. The first is the maximum extent of the plasma over the surface of the dielectric. The results from the model and experiments are show in Figure 9. Also included in the figure are experimental results from Enloe et al. ${ }^{33}$ The figure shows the extent of the plasma as a function of the peak-to-peak voltage. First, we notice that the plasma extent varies varies approximately linearly with the input voltage. Second, we observed very good agreement between the model results and the experimental results. The agreement with our experiment is better because the model used a dielectric coefficient for PC board material (glassepoxy) which was used in our experiments. The experiments by Enloe et al. ${ }^{33}$ used Kapton film which has a different dielectric coefficient and thickness.

This result is very important for the design of plasma actuator. Post ${ }^{26}$ noted that at higher voltages, if the width of the covered electrode was too small, the magnitude of the induced velocity would asymptote. A similar result was found for the thrust produced by the actuator in experiments by Enloe et al. ${ }^{33}$

Another distinctive feature that can be compared is the sweep out velocity. This corresponds to the space-time slope of the outer edge of the light intensity contours. A comparison for the plasma sweep velocity between the space-time simulations and plasma illumination experiments are shown in Figure 10. Also included in the figure are results from the experiments of Enloe et al. ${ }^{33}$ The simulation shows that the plasma sweep velocity increases slightly with the applied voltage amplitude. The agreement with the experiment is very good. The range of sweep velocities is from 75 to $110 \mathrm{~m} / \mathrm{s}$.

Another set of numerical simulation has been performed to study the dependence of the major plasma characteristics, such as the maximum plasma extent over the surface of the dielectric and the plasma sweep velocity, on the applied a.c. frequency. These results were again compared to the experimental results. The comparison between the experimental results and the space-time lumped-element circuit model simulation are presented in Figures 11 and 12. As it can be seen in these figures, the model predicts that the maximum plasma extent over the encapsulated electrode does not increase with the increasing a.c. frequency. This 


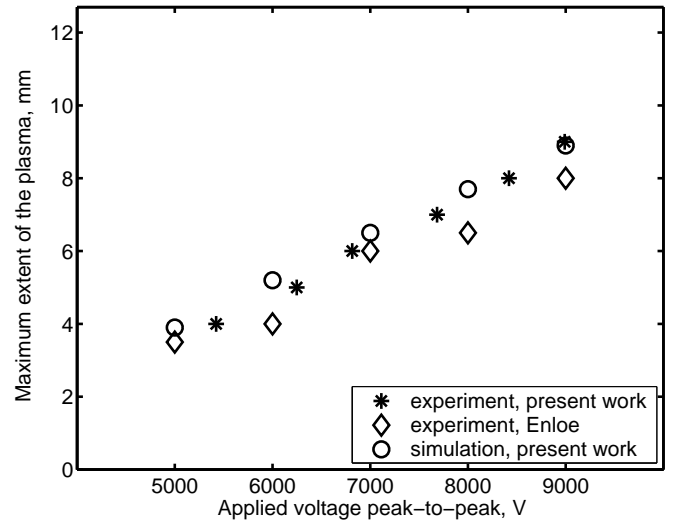

Figure 9. Comparison between space-time model and experiments for the maximum plasma extent over covered electrode as function of voltage.

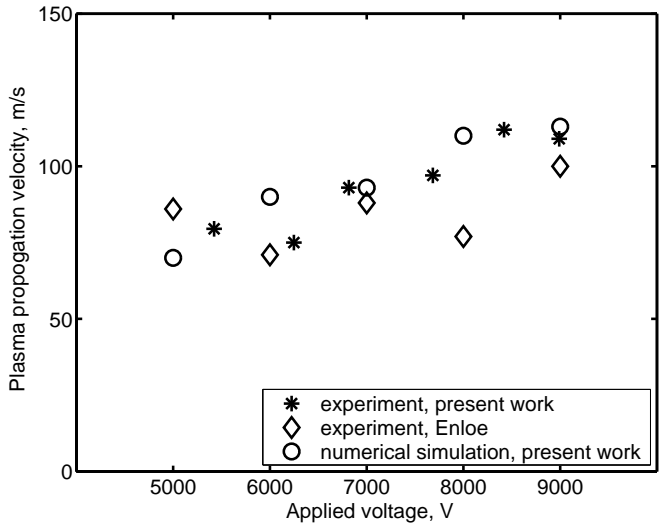

Figure 10. Comparison between space-time model and experiment for plasma sweep velocity as function of voltage.

also means that the plasma propagation velocity would increase linearly with the applied frequency. This is consistent with the experimental observations. The results obtained from the numerical simulation for the plasma sweep velocity are in the range of $75-200 \mathrm{~m} / \mathrm{s}$ and match the experimental result very well.

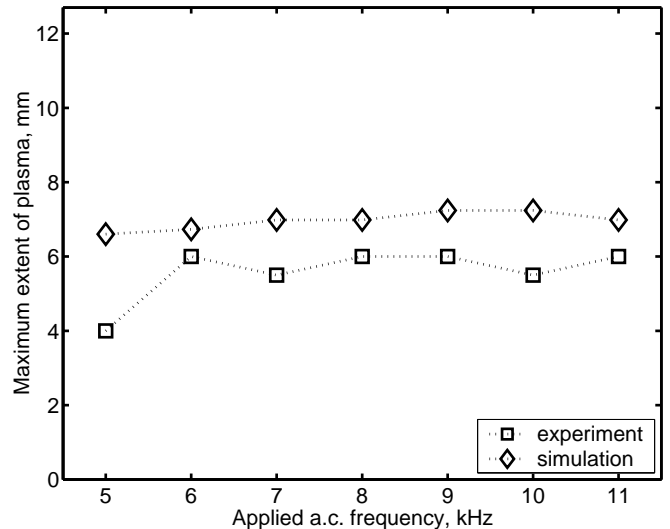

Figure 11. Comparison between space-time model and experiment for maximum extent of the plasma as function of applied a.c. frequency.

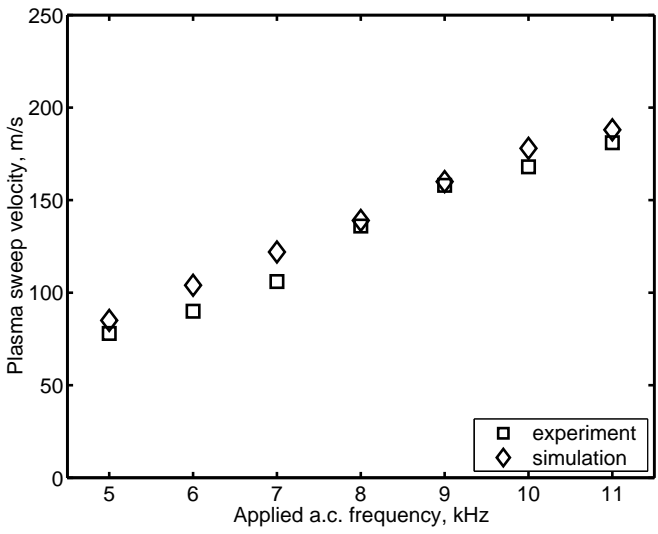

Figure 12. Comparison between space-time model and experiment for plasma sweep velocity as function of applied a.c. frequency.

These results are necessary to obtain the plasma body force. The solution for the voltage on the surface of the dielectric serves as the time-dependent boundary condition of the electric potential, $\varphi$, in the electrostatic equation (6) at the electrodes. The extent of the plasma on the surface of the dielectric specifies the region of the presence of charges above the encapsulated electrode, as shown in Figure 13. The value of the electric potential on the exposed electrode are specified to be the applied voltage $V_{a p p}(t)$. The boundary conditions at the outer boundaries are set to the "infinity" boundary conditions $(\varphi=0)$. With these boundary conditions, the electrostatic equation

$$
\nabla(\varepsilon \nabla \varphi)=\frac{1}{\lambda_{D}^{2}} \varphi
$$

is solved. This is done at each time step of the lumped-element circuit model to account for the time dependence of the ionization. The solution of the electrostatic equation is then used to calculate the timedependent body force produced by the plasma, given as before as

$$
\overrightarrow{f_{b}^{*}}=\rho_{c} \vec{E}=-\left(\frac{\varepsilon_{0}}{\lambda_{D}^{2}}\right) \varphi \vec{E} .
$$




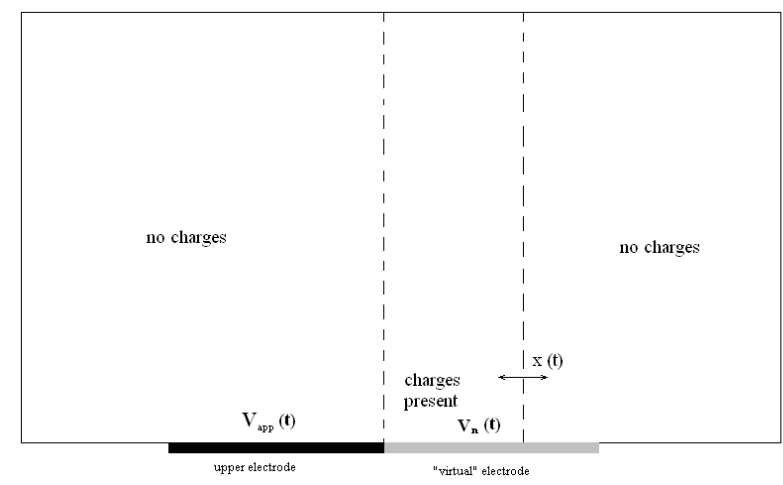

Figure 13. Computational domain for calculation of unsteady plasma body force.

An example of the normalized magnitude of the plasma body force for the asymmetric electrode arrangement illustrated in Figure 13 is shown in Figure 14. This shows the body force distribution in space and time. It can be noticed that the largest magnitude of the plasma body force is near the edge of the exposed electrode. From that location the magnitude decays rapidly over the surface of the dielectric. Recall that experiments indicated that the plasma illumination decreased exponentially. This is consistent with the decay that comes from the solution of the lumped-element circuit model. It justifies the exponential weighting that was used in the electrostatic models developed earlier.

Another feature that can be noticed from Figure 14 is that the body force peaks four times during one a.c. cycle. The fact that the body force has four peaks results from the body force formulation (7) and the fact that during the a.c. cycle the electric potential, $\varphi$, is equal to 0 when $V_{a p p}=0$, and the electric field, $\vec{E}$ is equal to 0 when applied voltage reaches its maximum and minimum values.

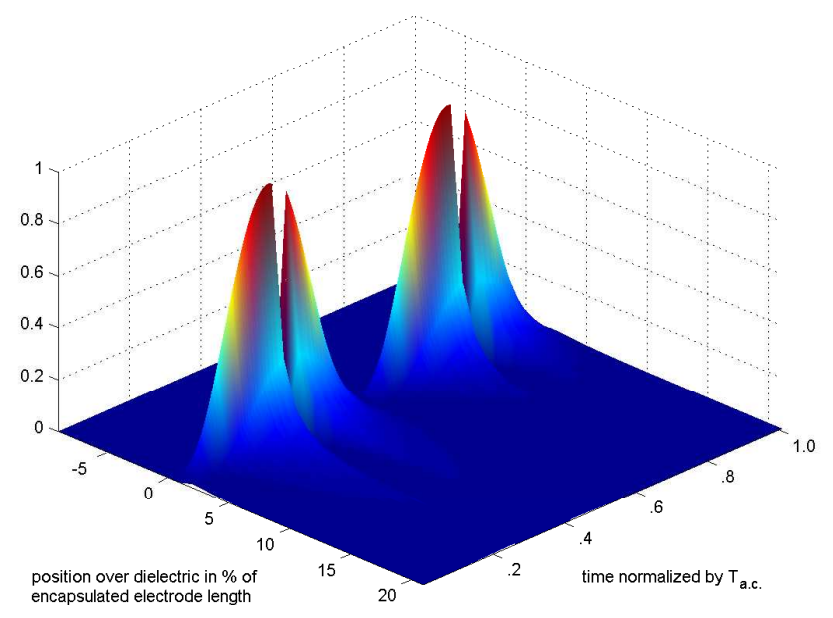

Figure 14. Normalized plasma body force magnitude as function of time and position over covered electrode.

Another important feature of the plasma body force is the direction of the force vector during the A.C. cycle. To illustrate this, the body force vectors and amplitudes have been plotted at two time locations corresponding to two peaks in the body force at $t=0.2 \cdot T_{\text {a.c. }}$ and $t=0.7 \cdot T_{\text {a.c. }}$. This is shown in Figures 15 - 16. This illustrates that the actuator force vector is always in one direction, from the exposed electrode towards the covered electrode. Because the body force magnitude peaks four times within the a.c. period and two of the peaks are slightly smaller, this might be represented as "Push-push-Push-push" for one a.c. period

These observations suggest that the spectral analysis of the body force may reveal important features of the plasma discharge and also serve as a tool for comparison with the experimental data. For example, the spectrum results can be directly compared to the acoustic or accelerometer measurements of the plasma 

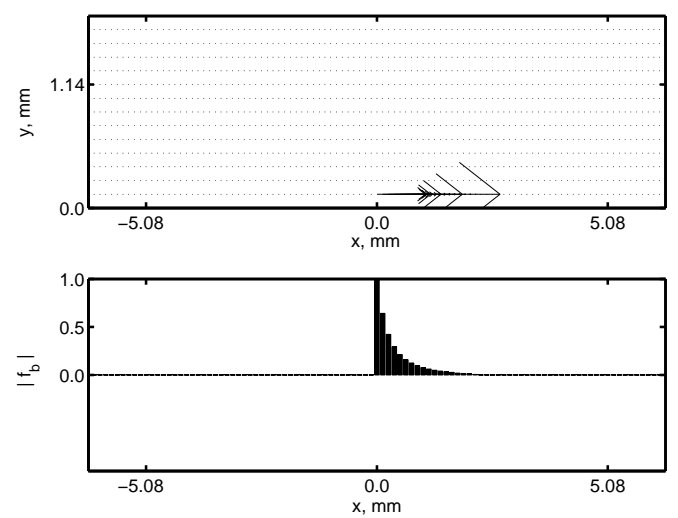

Figure 15. Plasma body force vector field and body force amplitude at $t=0.2 \cdot T_{a . c .}$. The body force is normalized by the maximum value in the a.c. cycle.
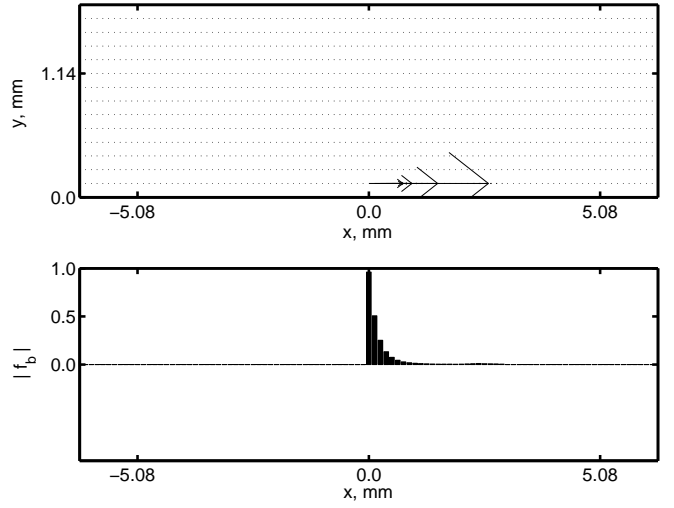

Figure 16. Plasma body force vector field at $t=0.7 \cdot T_{a . c .}$. The body force is normalized by the maximum value in the a.c. cycle.

actuator. ${ }^{34}$

We performed the spectral analysis of the plasma body force signal shown previously in Figure 14. The resulting spectrum is shown in Figure 17. it can be noticed that the dominating frequency in this spectrum is twice the frequency of the plasma actuator. This result is consistent with the body force signal shown in Figure 14 where the both peaks of the body force are approximately equal. This domination of the second harmonic was also noticed in the experiments by Porter et al. ${ }^{34}$ In their experiment, the spectrum of the accelerometer signal showed the dominance of the second harmonic in one of the studied cases $(7 \mathrm{kHz})$, but not in the other two cases ( 5 and $6 \mathrm{kHz}$ ). This difference in their observations may be explained by the transfer to the filamentary regime at lower frequencies when the plasma becomes non-uniform.

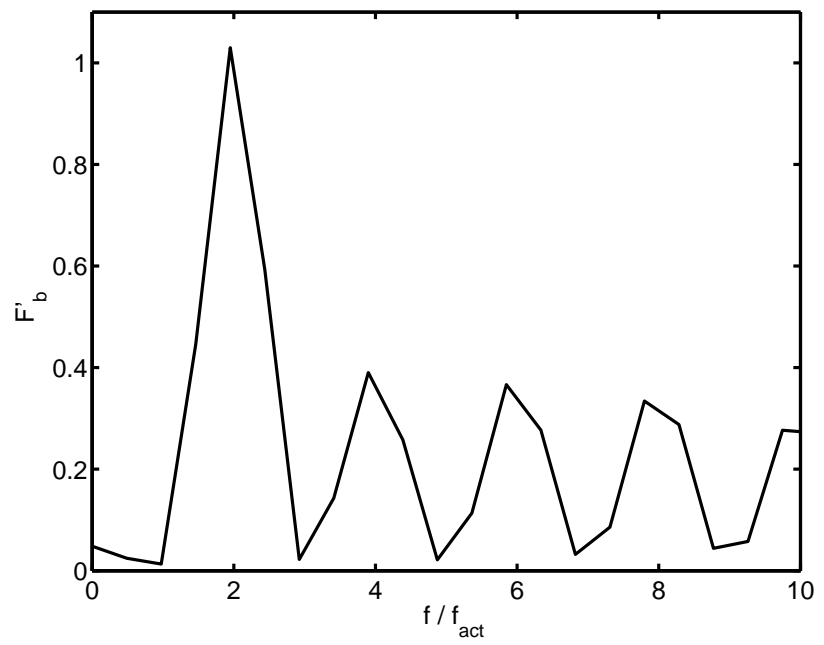

Figure 17. Spectrum of the plasma body force obtained with space-time lumped-element circuit model.

Using the space-time lumped-element circuit model we were able to study the effect that different dielectric materials had on the plasma body force. Three different materials were tested with the dielectric coefficients $\varepsilon=10,100$, and 1000. A set of numerical simulations has been carried out for a range of a.c. frequencies from $1 \mathrm{~Hz}$ to $10 \mathrm{kHz}$. The results of these simulations are presented in Figures 18 and 19 for the plasma body force and the power dissipated by the actuator.

From the body force results we notice that for each materials examined there exists the optimum frequency at which the body force magnitude is a maximum. This result is consistent with the general theory of the $\mathrm{RC}$ circuits. On the other hand, we note that the dissipated power increases with the a.c. frequency. This indicates that the optimum performance of the actuator requires the proper selection of the driving a.c. 
frequency. Off-optimum operation will result in excessive ohmic heating.

The optimum frequency of the plasma actuator depends on the dielectric properties of the material, or more specifically on the bulk capacitance of the actuator. The capacitance is proportional to $\varepsilon / h$, where $h$ is the thickness of the dielectric. In the example, only the dielectric coefficient was changed to change its capacitance. I could have been changed in like manner through the thickness of the dielectric or in combination with $\varepsilon$. For our large range of values, the optimum body force frequency changed from 10 to $1000 \mathrm{~Hz}$. For Kapton with $\varepsilon=3.3$, and our typical thicknesses of 0.006 in., the optimum frequency is in the range of $5 \mathrm{kHz}$.

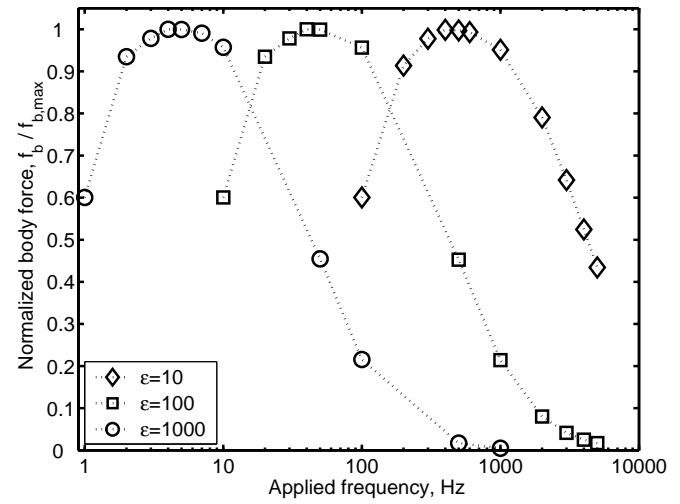

Figure 18. Effect of dielectric material on plasma body force.

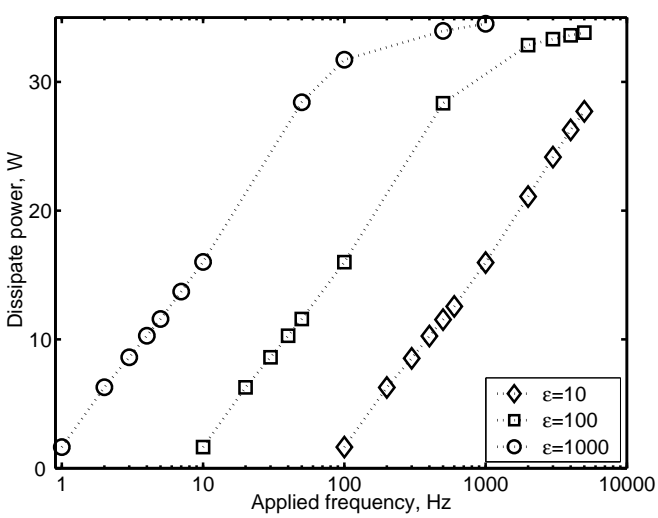

Figure 19. Effect of dielectric material on power dissipated by the plasma actuator.

The space-time lumped-element circuit model showed excellent agreement with the experimental results. It allowed us to calculate the time-dependent volume of the plasma over the electrodes during the a.c. cycle. It also provided an efficient manner for calculating the plasma body force that could be easily incorporated into the Navier-Stokes flow solvers.

\section{Leading-edge Separation Control using SDBD Plasma Actuators}

The maximum lift and stall characteristics of a wing affect many performance aspects of aircraft including take-off and landing distance, maximum and sustained turn rates, climb and glide rates, and flight ceiling. ${ }^{35}$ In a 2-D wing, the maximum achievable lift is ultimately limited by the ability of the flow to follow the curvature of the airfoil which affects the pressure gradient. When the pressure gradient becomes too adverse, the flow separates. In many cases at extreme angles of attack, this occurs at the leading edge.

One approach to prevent leading-edge separation is to increase the leading edge radius. This is the principle effect of a leading edge flap. An example is a Krueger flap, which consists of a hinged surface on the lower side of the wing that can extend out and ahead of the wing leading edge. A slotted leading-edge flap (slat) is the leading-edge equivalent of the trailing-edge slotted flap. It works by allowing air from the high-pressure lower surface to flow to the upper surface to add momentum to the boundary layer to overcome an adverse pressure gradient, and prevent flow separation.

Conventional multi-element wings and wings with movable control surfaces such as the leading-edge slats contain gap regions that are a major source of airframe noise and unsteady structural loading, especially at high deflection angles. Most of the noise originates from the separated flow in the gap regions. It is also known that the hinge gaps contribute to as much as $10 \%$ of the form drag component of the viscous drag on the wing. ${ }^{35}$ In order to improve the aerodynamic performance of the wing, it is desirable to either completely replace the traditional moving surfaces with hingeless control surfaces, or limit the deflections of moving surfaces without compromising the wing's performance. Both these alternatives necessitate other approaches for controlling flow separation over the surface of the wing. The following simulation addresses one such approach by using a single-dielectric barrier discharge (SDBD) plasma actuator to control leadingedge flow separation on the wing in a manner that might potentially replace leading-edge slats. 


\section{III.A. Problem Formulation}

The numerical simulation was performed on a NACA 0021 airfoil with chord length of $.3048 \mathrm{~m}$. This was intended to match an experiment on a NACA 0021 airfoil with a plasma actuator on the leading edge. The experiment investigated a range of free-stream speeds from 10 to $30 \mathrm{~m} / \mathrm{s}$ giving a range of chord Reynolds numbers of $0.205 \times 10^{6}$ to $0.615 \times 10^{6}$. Lift and drag measurements in the experiment were measured using a force balance. Lift and drag coefficients were measured for a range of angles of attack from 0 to 25 degrees. Therefore these experimental results provide a basis for comparison to the numerical simulations.

The first step in the simulations was to compute the body force produced by the actuator at the leading edge. For this the electro-static model was used. As it was pointed out earlier, the electro-static model does not include the spatial variation of the charged air over the dielectric-covered electrode. However we can compensate for that effect by adding an experimental decay weighting of the body force. The experimental spatial weighting agreed well with experimental observations of the plasma light illumination presented in Figure 8, and the space-time simulations presented in Figure 14.

Using the electro-static SDBD actuator model, the body force computations were performed on an unstructured grid using COMSOL Multiphysics (FemLab). ${ }^{36}$ The center line of the actuator was located at the leading edge $(x / C=0)$. A schematic of the actuator arrangement on the airfoil is shown in Figure 20. The exposed actuator was located on the pressure side of the airfoil, and the embedded electrode was placed on the suction side. In order to represent the experiment, the electrodes were $1 / 2 \mathrm{in}$. wide. The dielectric material was a thick layer of a 3 mil Kapton. For the simulation, the grid was manually refined near the location of the actuator.

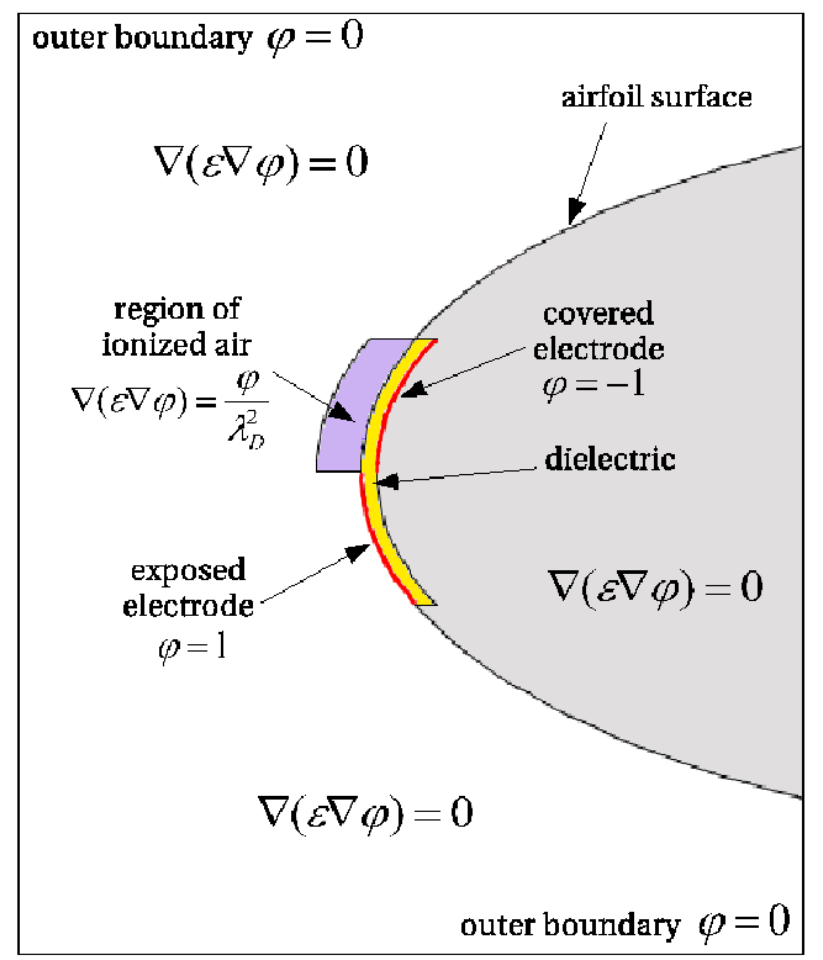

Figure 20. Schematic of the plasma actuator on the leading edge of NACA 0021 airfoil for body force computations.

The boundary conditions were set on the electrodes. This was a static electric potential equal to the applied voltage to the electrodes of $5 k V_{p-p}$. The electric potential far from the electrodes at the boundaries of the computational domain was set to zero. As in our previous body force calculations, the plasma was taken to be only over the dielectric that covers the electrode. The governing equation for the region over the exposed electrode was Laplace equation given as

$$
\nabla(\varepsilon \nabla \varphi)=0 .
$$


The governing equation for the region over the covered electrode was Poisson's equation given as

$$
\nabla(\varepsilon \nabla \varphi)=\frac{\varphi}{\lambda_{d}^{2}}
$$

The solution gives the body force magnitude and vector directions. These are shown in Figure 21. In this case, the body force values were taken from the unstructured grid and interpolated onto a structured grid used in the solution of the flow field. For this, the experimental spatial weighting has been applied.

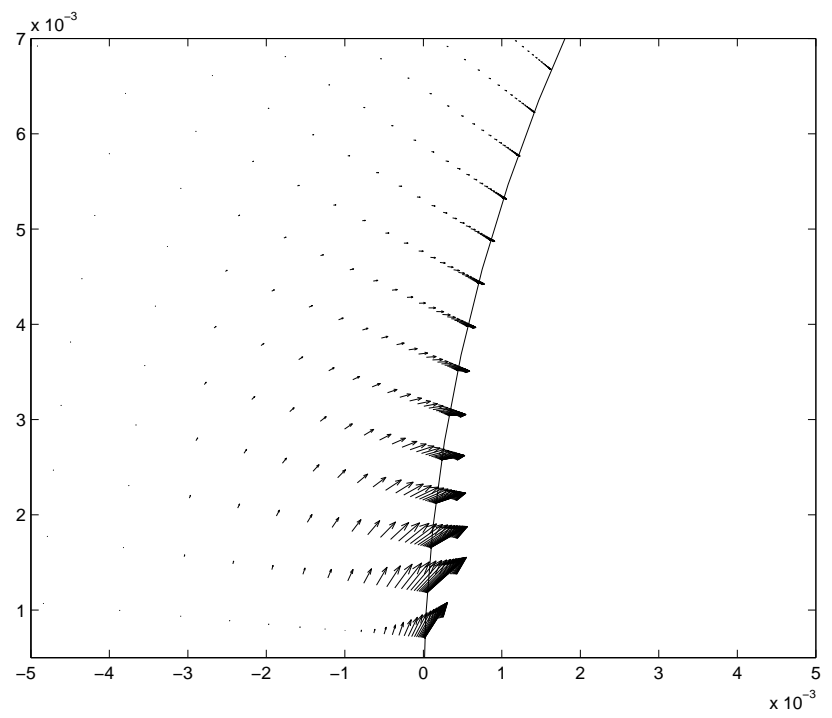

Figure 21. Computed steady plasma body force vectors near leading edge of NACA 0021 airfoil, shown on structured computational grid used for flow solver.

The flow simulation was performed using FLUENT. The structured $C$-type grid was meshed around the airfoil using GAMBIT. ${ }^{37}$ The surface of the airfoil was meshed with 400 grid points. There were 100 grid points used in the direction normal to airfoil surface, with a majority of the grid points located in the region of the boundary layer. The inlet boundary was located 10 chord lengths from the airfoil leading edge, and the outflow boundary was located 20 chord lengths downstream the airfoil trailing edge. A zoomed-in view of the computational grid used in the flow simulations is shown in Figure 22.

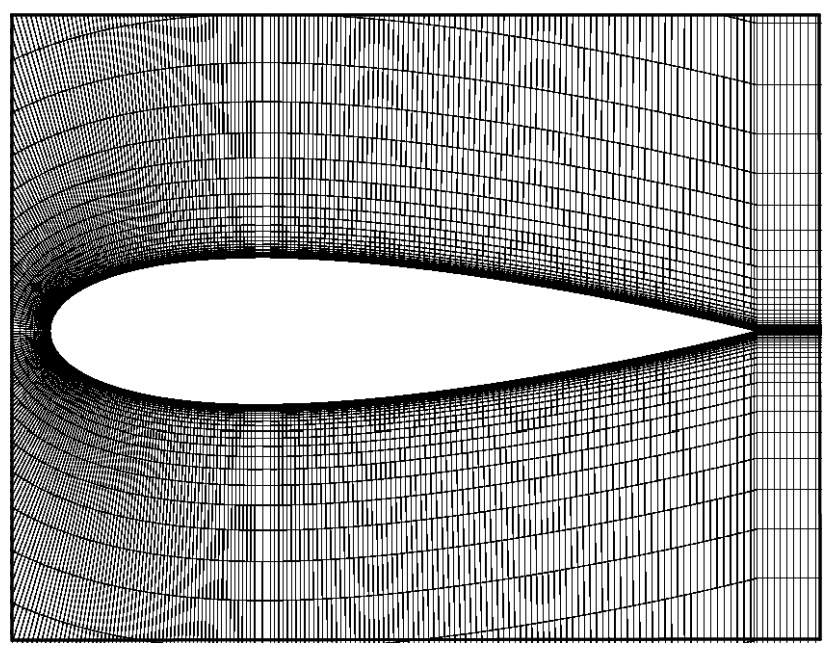

Figure 22. Zoomed-in view of computational grid showing grid point clustering in the region of the boundary layer.

The governing model equations for the flow were 2-D unsteady Reynolds-averaged Navier-Stokes (RANS) equations. Our intention was that the flow be incompressible. 
The standard boundary conditions were used. They induced no-slip and no-penetration on the airfoil surface. Pressure far-field conditions are used in FLUENT to model the free-stream condition at infinity. This is where the free-stream Mach number and static conditions were specified. The pressure far-field boundary condition is a non-reflecting boundary condition based on the introduction of Riemann invariants for a one-dimensional flow normal to the boundary. For the present simulation, the inflow was set at $35 \mathrm{~m} / \mathrm{s}$ (corresponding to $M=0.1$ ). The angle of attack was varied by changing the angle of the flow at the inflow boundary. The turbulence parameters were defined at the far-field boundary in terms of the turbulence intensity and turbulence viscosity ratio. The turbulence intensity of the inflow was set to $0.1 \%$. This value corresponds to the maximum value of the turbulence intensity in the experiments. The turbulence viscosity ratio was set to be 10. An additional equation for energy conservation was included in formulation.

A second order implicit formulation was used for time and space discretizations. The SIMPLE algorithm was used for the pressure-velocity coupling. This algorithm uses a relationship between velocity and pressure corrections to enforce mass conservation and to obtain the pressure field. In the SIMPLE algorithm, an approximation of the velocity field is obtained by solving the momentum equations. The pressure gradient term is calculated using the pressure distribution from the previous iteration or an initial guess. The pressure equation is formulated and solved in order to obtain the new pressure distribution. From this the velocities are corrected and a new set of conservative fluxes are calculated. A more detailed description of the SIMPLE algorithm may be found in the Fluent documentation files. ${ }^{38}$

The first simulation was performed at 0 degrees angle of attack. The flow was initialized from the inflow boundary. Afterwards, the solution at higher angles of attack used the previous converged solution at one degree smaller as the initial condition for the simulation.

The identification of vortical structures has been performed using the $-\lambda_{2}$ technique developed by Hussain. ${ }^{39}$ In this technique, the eigenvalues of the symmetric tensor $S^{2}+\Omega^{2}$ are considered: here $S$ and $\Omega$ are the symmetric and antisymmetric parts of the velocity gradient tensor $\nabla u$. The vortex region is identified by the negative values of the second eigenvalue, $\lambda_{2}$.

In planar flow, the velocity gradient can be written in general form as

$$
\nabla u=\left(\begin{array}{cc}
a & b \\
c & -a
\end{array}\right),
$$

where $a=u_{x}, b=u_{y}$ and $c=v_{x}$. Therefore, we get that the $S^{2}+\Omega^{2}$ matrix in this case would be given as

$$
\nabla u=\left(\begin{array}{cc}
a^{2}+b c & 0 \\
0 & a^{2}+b c
\end{array}\right),
$$

and the characteristic equation can be written as

$$
\left(a^{2}+b c-\lambda\right)\left(a^{2}+b c-\lambda\right)=0 .
$$

This equation has the solution

$$
\lambda_{1,2}=a^{2}+b c
$$

Thus, negative $\lambda_{2}$ requires that

$$
u_{x}^{2}+u_{y} v_{x}<0
$$

\section{III.B. Results of Leading-edge Separation Control}

During the first phase of the simulations, the base flow without flow control was modeled. The behavior of the lift and drag coefficients was taken to be the convergence criteria for the flow problem.

As the simulations showed, at lower angles of attack, below approximately 12 degrees, the linear airfoil theory holds and the slope, $\frac{d C_{L}}{d \alpha}=2 \pi$. Therefore at least in this region the solution is known. In addition, the experimental results for this airfoil gave an indication of the stall angle of attack.

The $v^{2}-f$ turbulence model was used in these simulations. This model has been shown to predict the boundary layer separation correctly. ${ }^{40}$ Our calculations performed with the $v^{2}-f$ turbulence model showed a decrease in lift and an increase in drag at 18 degrees. This agreed best with the experiment. Based on these tests, the $v^{2}-f$ turbulence model was used for all of other calculations with and without the plasma actuators. 
Figure 33 documents the baseline computations for the NACA 0021 airfoil. This shows the expected lift coefficient slope of 0.11 degrees $^{-1}$. The maximum lift coefficient for this airfoil is approximately 1.2 . The airfoil stalls at approximately $18^{\circ}$ angle of attack. This simulation is reasonably close to this value, although the drop-off in lift is not as sharp as in the experiment. It should be pointed out that the experimental values were not compensated for blockage effects. Blockage effects tend to increase slightly the lift coefficient slope.

For the second part of the simulation, a steady actuator effect was included in the governing equations. As described earlier,the plasma actuator was located at the leading edge of the airfoil. With FemLab, the plasma body force was introduced using the User-Defined Function (UDF) Module. For the steady actuator, the body force was constant in time. That is its magnitude was the same at each time step of the calculations.

The third part of the simulation dealt with "unsteady" plasma actuation. The previous experimental work $^{27}$ has indicated that better flow re-attachment would occur by operating the actuator at a periodic frequency. The optimum frequency was found in experiments to correspond to $F^{+}=f c / U_{f s}=1$. An example of the unsteady operation used in the experiment is shown in Figure 23. This switches on and off the a.c. carrier that ionizes the air in the experiments at a prescribed frequency and duty cycle. The experiments found that a $10 \%$ duty cycle was effective.
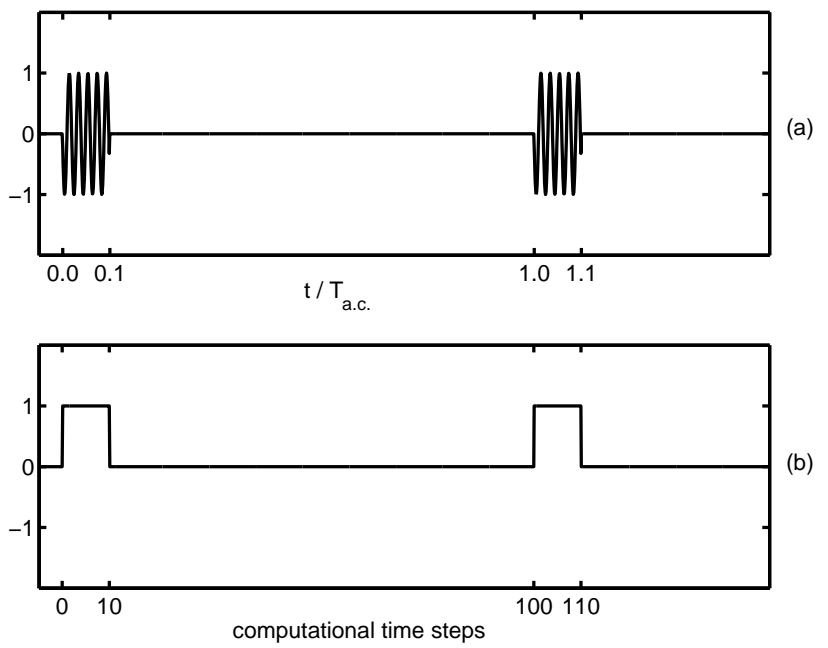

Figure 23. Example of short duty cycle a.c. input for unsteady operation of plasma actuators (a) and its numerical representation (b).

In the simulations, the body force was represented in the discrete computational time steps of the time dependent formulation. For example, if 100 time steps represented the full period of the actuator at $F^{+}=1$, for a $10 \%$ duty cycle, the body force would be non-zero for 10 time steps, and zero for the other 90 time steps. This was implemented using the UDF Module just like with the steady actuator effect.

A sample test case was performed in the still air $\left(U_{f s}=0 \mathrm{~m} / \mathrm{s}\right)$ to illustrate the effect of the plasma actuator presence on the airfoil surface. For this, the actuator was impulsively switched on, and maintained for a long time period. Velocity vectors recorded a short time after the actuator was started are shown in Figure 24. Note that this is similar to the simulations that were done previously. The impulsively started actuator produced a local wall jetting effect and a "starting" vortex that is turning counterclockwise. Figures 25 to 27 shows the vector field $0.02,0.06$ and 0.18 seconds later, respectively. This shows the flow sweeping over the leading edge of the airfoil. There is still a counter-clockwise circulation associated with the impulsive start of the actuator. The wall jet magnitude in this simulation reached a maximum value of $8.37 \mathrm{~m} / \mathrm{s}$.

An example of the effect of the plasma actuator on the flow over the airfoil at a post-stall angle of attack of 23 degrees is considered. The flow over the airfoil without any actuation is shown in Figure 30. This shows a large separation bubble that starts from the leading edge and extends past the trailing edge. The streamlines show a large circulation indicating flow reversal over the airfoil and into the wake. This is supported by the $\lambda_{2}=0$ contours which show the large separation structure. The streamlines look very much like the streamline photographs of similar airfoils at post-stall angles of attack such as those by Post. ${ }^{26}$

In contrast to the case with the actuator off, Figure 31 shows streamlines for the same angle of attack (23 degrees) with a steady plasma actuator on. This shows that the flow is attached at the leading edge, leaving 


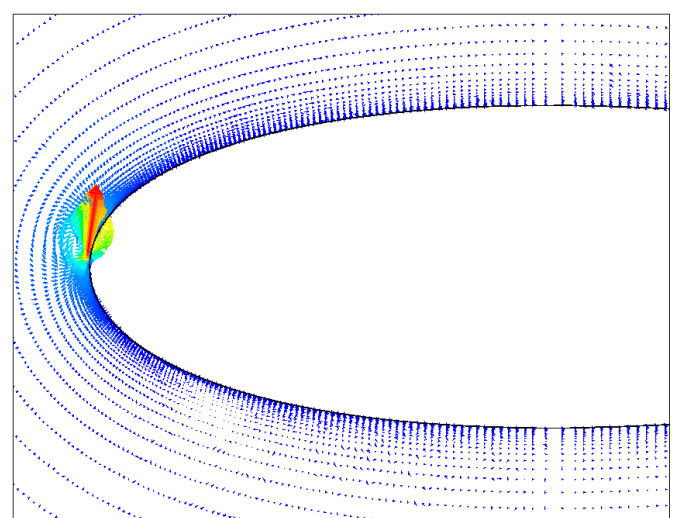

Figure 24. Velocity vector field near leading edge of airfoil at $t=0.00001$ seconds after the impulsive start of the actuator. The largest velocity vector corresponds to $|V|=4.03 \mathrm{~m} / \mathrm{s}$.

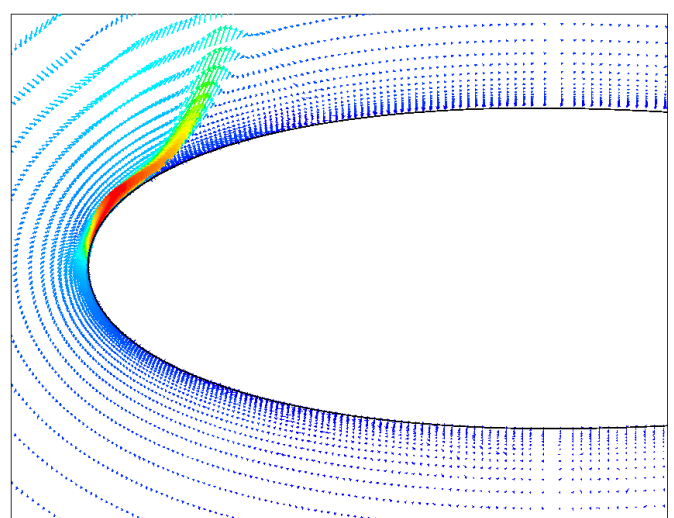

Figure 26. Velocity vector field near leading edge of airfoil at $t=0.06$ seconds after the impulsive start of the actuator. The largest velocity vector corresponds to $|V|=8.36 \mathrm{~m} / \mathrm{s}$.

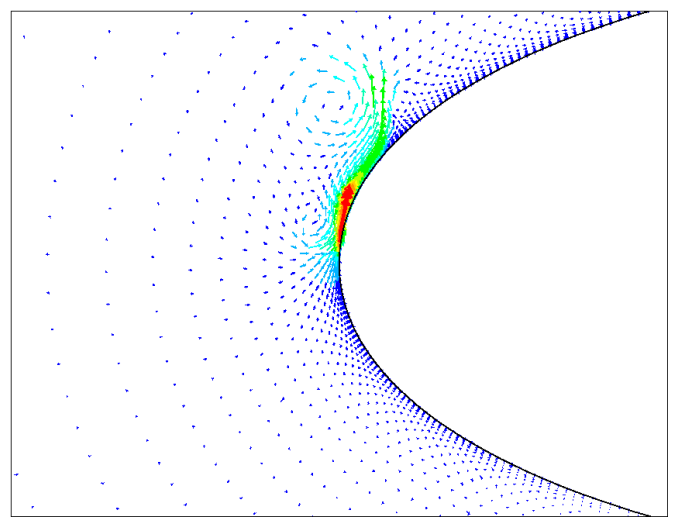

Figure 28. Velocity vector field at $t=0.01743$ seconds in still air. The plasma actuator is working in unsteady mode at $120 \mathrm{~Hz}$. The largest velocity vector corresponds to $|V|=$ $4.49 \mathrm{~m} / \mathrm{s}$.

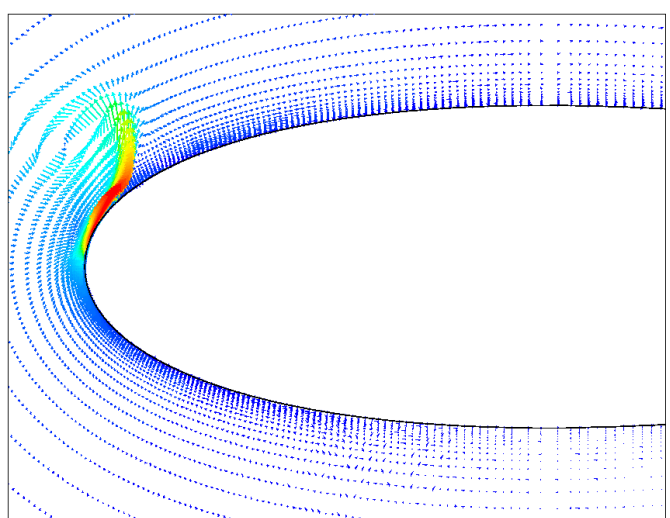

Figure 25. Velocity vector field near leading edge of airfoil at $t=0.02$ seconds after the impulsive start of the actuator. The largest velocity vector corresponds to $|V|=8.34 \mathrm{~m} / \mathrm{s}$.

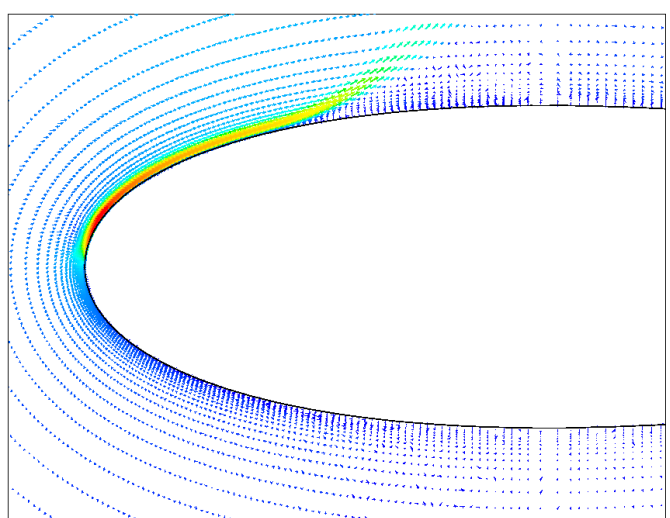

Figure 27. Velocity vector field near leading edge of airfoil at $t=0.18$ seconds after the impulsive start of the actuator. The largest velocity vector corresponds to $|V|=8.37 \mathrm{~m} / \mathrm{s}$.

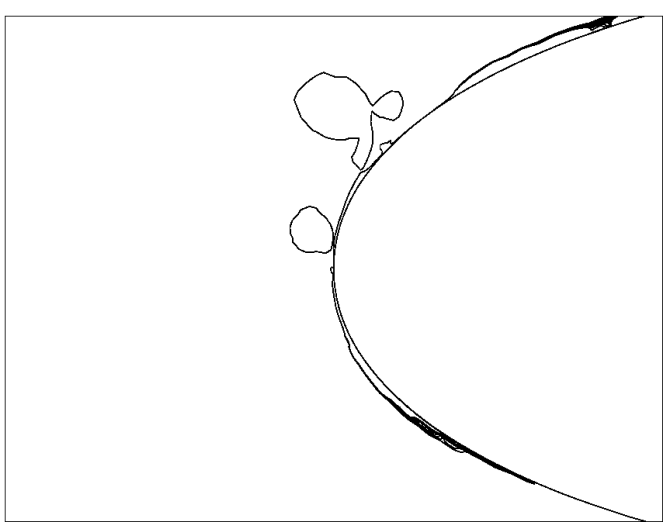

Figure 29. Contour lines of $\lambda_{2}=0$ (b) at $t=0.01743$ seconds in still air. The plasma actuator is working in unsteady mode at 120 Hz. 
only a smaller trailing edge separation. Visually, the wake of the airfoil is significantly smaller, which will translate into lower overall drag.

In case of the unsteady flow actuation, the flow is still attached at the leading edge of the airfoil as it is shown in Figure 32. The point were the boundary layer separates from the airfoil surface is approximately at the same location as in the steady actuation case. But the separation bubble appears to be shorter than the one during the steady actuation. This agrees well with the experimental observations.

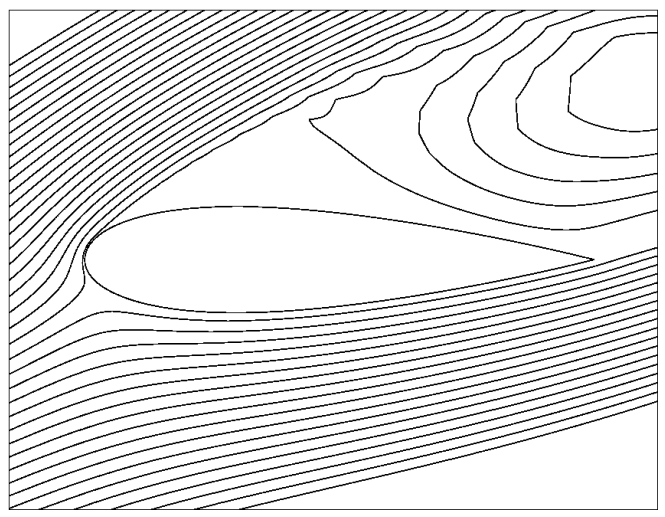

Figure 30. Contour lines of stream function, no actuation, 23 degrees angle of attack.

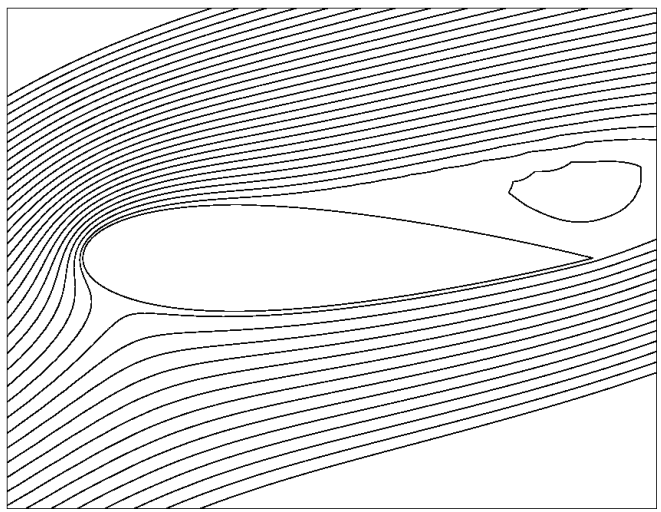

Figure 31. Contour lines of stream function for steady actuation, 23 degrees angle of attack.

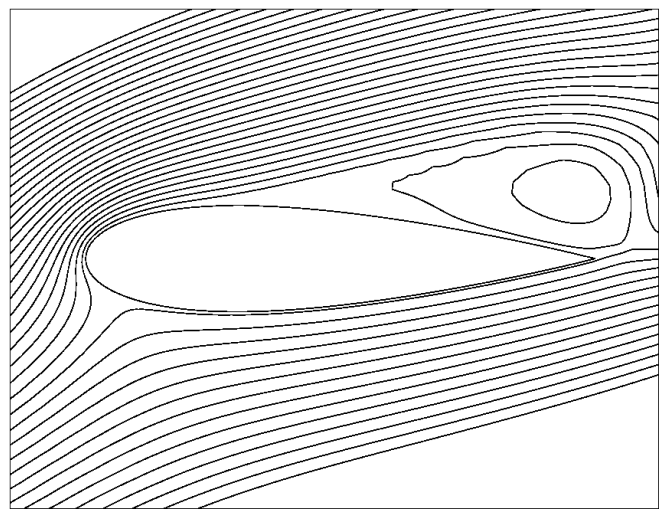

Figure 32. Contour lines of stream function for unsteady actuation at $120 \mathrm{~Hz}$, duty cycle of $10 \%, 23$ degrees angle of attack.

A series of simulations at a range of angles of attack with steady and unsteady plasma actuators were 
conducted. The results of these are summarized in terms of the lift coefficient versus angle of attack in Figure 33. In general both the steady and unsteady plasma actuators significantly increased the stall angle of attack. The unsteady actuation was at the optimal frequency of $F^{+}=1$.

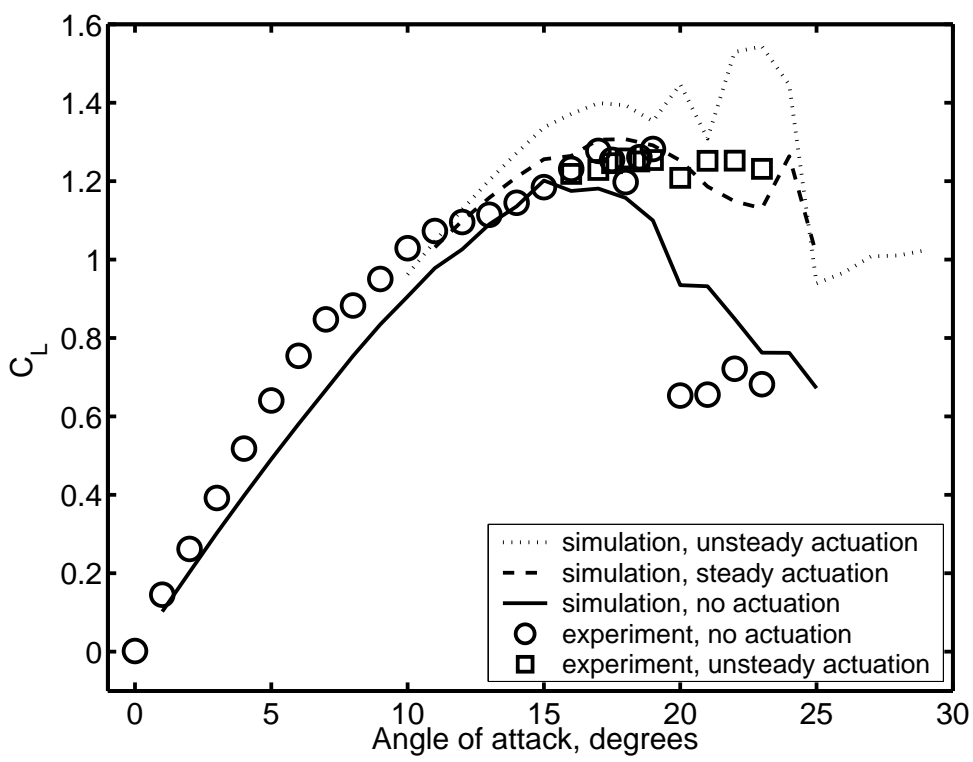

Figure 33. Comparison between numerical and experimental data for lift coefficient versus angle of attack for uncontrolled case, steady and unsteady actuation. Numerical simulations performed at $U_{f s}=35 \mathrm{~m} / \mathrm{s}$. Experiment performed at $U_{f s}=30 \mathrm{~m} / \mathrm{s}$.

The results of this simulation show good agreement with the experiments. The plasma actuator placed near the leading edge of the airfoil delays the separation and increases the stall angle of attack. The unsteady flow actuation shows even better results than the steady actuation improving the airfoil characteristics at high angles of attack.

\section{Conclusions}

To account for the change in the volume of plasma with respect to the applied voltage amplitude and frequency, the lumped-element circuit model was created. This model represented the plasma as a series of parallel networks, each consisting of resistive and capacitive elements, and zener diodes that controlled the values based on the current direction. The effect of the applied voltage amplitude and a.c. frequency on the plasma characteristics was examined with the space-time lumped-element circuit model. The results were found to be in good agreement with the experimental results. The results of space-time lumped-element circuit model were used to calculate the time-resolved plasma body force. It was found that the plasma body force has four peaks during the a.c. cycle, and that the direction of the plasma body force is the same all the time. The effect of the dielectric properties of the material was studied with the space-time lumped-element circuit model. It was shown that for each material tested there exists an optimal frequency at which the plasma body force is maximum.

The leading-edge separation control on NACA 0021 using single-dielectric barrier discharge plasma actuator was studied numerically. FLUENT software was used to solve two-dimensional unsteady Reynoldsaveraged Navier-Stokes equations with the $v^{2}-f$ turbulence model. The plasma actuator was modeled on the leading edge of the airfoil. It was found that the steady actuation increased the lift coefficient and delayed the stall by approximately 2 degrees of angle of attack. The unsteady actuation was shown to produce even better results. The stall angle in that case was increased by 5 degrees. These results are consistent with the experiments for the same conditions.

\section{Acknowledgment}

The authors are pleased to acknowledge support on the numerical simulation portion of this work to Orbital Research Inc. under an SBIR Phase II Contract that was monitored by Mr. Mehul Patel. The 
experimental work on the NACA 0021 airfoil reported here was supported by Boeing, Inc. and monitored by Mr. Joseph Silkey.

\section{References}

${ }^{1}$ Matlis, E. H., Controlled experiments on instabilities and transition to turbulence on a sharp cone at Mach 3.5, Ph.D. thesis, University of Notre Dame, 2004.

${ }^{2}$ Corke, T. C., Jumper, E. J., Post, M. L., Orlov, D., and McLaughlin, T. E., "Application of weakly-ionized plasmas as wing flow-control devices." AIAA Paper 2002-0350, 2002.

${ }^{3}$ Corke, T. C., Mertz, B., and Patel, M. P., "Plasma Flow Control Optimized Airfoil." AIAA Paper 2006-1208, 2006.

${ }^{4}$ Nelson, C., Cain, A., Patel, M., and Corke, T., "Simulation of Plasma Actuators Using the Wind-US Code." AIAA Paper 2006-634, 2006.

${ }^{5}$ Patel, M. P., Sowle, Z. H., Corke, T. C., and He, C., "Autonomous Sensing and Control of Wing Stall Using a Smart Plasma Slat." AIAA Paper 2006-1207, 2006.

${ }^{6}$ Huang, J., Documentation and control of flow separation on a linear cascade of Pak-B blades using plasma actuators., Ph.D. thesis, University of Notre Dame, 2005.

${ }^{7}$ Huang, J., Corke, T., and Thomas, F., "Separation control over low pressure turbine blades." Bulletin of the American Physical Society Division of Fluid Dynamics, Vol. 47, 2002.

${ }^{8}$ Huang, J., Corke, T., and Thomas, F., "Separation control over low pressure turbine blades." Bulletin of the American Physical Society Division of Fluid Dynamics, Vol. 48, 2003.

${ }^{9}$ Huang, J., Corke, T. C., and Thomas, F. O., "Plasma actuators for separation control of low pressure turbine blades." AIAA Paper 2003-1027, 2003.

${ }^{10}$ List, J., Byerley, A. R., McLaughlin, T. E., and VanDyken, R. D., "Using a plasma actuator to control laminar separation on a linear cascade turbine blade." AIAA Paper 2003-1026, 2003.

${ }^{11}$ Morris, S. C., Corke, T. C., VanNess, D., Stephens, J., and Douville, T., "Tip Clearance Control Using Plasma Actuators." AIAA Paper 2005-0782, 2005.

${ }^{12}$ Douville, T., Stephens, J., Corke, T., and Morris, S., "Turbine Blade Tip Leakage Flow Control by Partial Squealer Tip and Plasma Actuators." AIAA Paper 2006-20, 2006.

${ }^{13}$ Van Ness, D. K., Corke, T. C., and Morris, S. C., "Turbine Tip Clearance Flow Control using Plasma Actuators." AIAA Paper 2006-21, 2006. 2006.

${ }^{14}$ Thomas, F. O., Kozlov, A., and Corke, T. C., "Plasma Actuators for Bluff Body Flow Control." AIAA Paper 2006-2845,

${ }^{15}$ Asghar, A., Jumper, E. J., and Corke, T. C., "On the Use of Reynolds Number as the Scaling Parameter for the Performance of Plasma Actuator in a Weakly Compressible Flow." AIAA Paper 2006-21, 2006.

${ }^{16}$ Wilkinson, S. P., "Investigation of an Oscillating Surface Plasma for Turbulent Drag Reduction." AIAA Paper 2003-1023, 2003.

${ }^{17}$ Hultgren, L. S. and Ashpis, D. E., "Demonstration of separation delay with glow-discharge plasma actuators." AIAA Paper 2003-1025, 2003.

${ }^{18}$ Orlov, D., Corke, T., and Haddad, O., "DNS Modeling of Plasma Array Flow Actuators." Bulletin of the American Physical Society Division of Fluid Dynamics, Vol. 48, 2003.

${ }^{19}$ Orlov, D., Corke, T., and Post, M., "DNS Modeling of Plasma Array Flow Actuators." Bulletin of the American Physical Society Division of Fluid Dynamics, Vol. 47, 2002.

${ }^{20}$ Orlov, D., Erturk, E., Post, M., and Corke, T., "DNS Modeling of Plasma Array Flow Actuators." Bulletin of the American Physical Society Division of Fluid Dynamics, Vol. 46, 2001.

${ }^{21}$ Visbal, M. R. and Gaitonde, D. V., "Control of Vortical Flows Using Simulated Plasma Actuators." AIAA Paper 2006-505, 2006.

${ }^{22}$ Post, M. and Corke, T., "Separation control using plasma actuators." Bulletin of the American Physical Society Division of Fluid Dynamics, Vol. 47, 2002.

${ }^{23}$ Post, M. and Corke, T., "Airfoil leading-edge separation control using plasma actuators." Bulletin of the American Physical Society Division of Fluid Dynamics, Vol. 48, 2003.

${ }^{24}$ Post, M. L. and Corke, T. C., "Separation control on high angle of attack airfoil using plasma actuator." AIAA Paper 2003-1024, 2003.

${ }^{25}$ Post, M. L. and Corke, T. C., "Separation control using plasma actuators - stationary and oscillatory airfoils." AIAA Paper 2004-0841, 2004.

${ }^{26}$ Post, M. L., Plasma actuators for separation control on stationary and unstationary airfoils., Ph.D. thesis, University of Notre Dame, 2004.

${ }^{27}$ Corke, T. C., He, C., and Patel, M., "Plasma flaps and slats: an application of weakly-ionized plasma actuators." AIAA Paper 2004-2127, 2004.

${ }^{28}$ Enloe, C., McLaughlin, T., VanDyken, R., and Fuscher, J., "Plasma Structure in the Aerodynamic Plasma Actuator." AIAA Paper 2004-0844, 2004.

${ }^{29}$ Orlov, D. M., Modelling and Simulation of Single Dielectric Barrier Discharge Plasma Actuators., Ph.D. thesis, University of Notre Dame, 2006.

${ }^{30}$ Orlov, D. and Corke, T., "Numerical Simulation of Aerodynamic Plasma Actuator Effects." AIAA Paper 2005-1083, 2005. 
${ }^{31}$ Orlov, D., Corke, T., and Patel, M., "Electric Circuit Model for Aerodynamic Plasma Actuator." AIAA Paper 2006-1206, 2006.

${ }^{32}$ Enloe, L., McLaughlin, T., VanDyken, Kachner, Jumper, E., and Corke, T., "Mechanisms and Responses odf a SingleDielectric Barrier Plasma Actuator: Plasma Morphology." AIAA, Vol. 42, 2004, pp. 589-594.

${ }^{33}$ Enloe, L., McLaughlin, T., VanDyken, Kachner, Jumper, E., Corke, T., Post, M., and Haddad, O., "Mechanisms and Responses odf a Single-Dielectric Barrier Plasma Actuator: Geometric Effects." AIAA, Vol. 42, 2004, pp. 585-604.

${ }^{34}$ Porter, C., Baughn, J., McLaughlin, T., Enloe, C., and Font, G., "Temporal Force Measurements on an Aerodynamic Plasma Actuator." AIAA Paper 2006-104, 2006.

${ }^{35}$ Corke, T. C., Design of Aircraft, Prentice Hall Publishers, New York, 2002.

${ }^{36}$ COMSOL Multiphysics 3.2 User's Guide, 2005.

${ }^{37}$ Gambit 2.2.30 Documentation, 2005.

${ }^{38}$ FLUENT 6.2 Documentation, 2005.

${ }^{39}$ Hussain, F. and Jeong, J., "On the identification of a vortex." Journal of Fluid Mechanics, Vol. 285, 1995, pp. 69-94.

${ }^{40}$ Durbin., P., "Separated Flow Computations with the $k-\epsilon-v^{2}$ Model." AIAA Journal, Vol. 33, 1995, pp. 659-664. 